\title{
The Factors Influencing the Naming Practice in the Algerian Society
}

\author{
Samia Azieb ${ }^{1, ~}$, Mahmoud Qudah ${ }^{2}$ \\ ${ }^{1}$ Department of English Language and Literature, University of Jordan, Amman, Jordan \\ ${ }^{2}$ Department of Humanities, Princess Sumaya University for Technology, Amman, Jordan \\ Email address: \\ mq@psut.edu.jo(M. Qudah), aziebsamia@yahoo.com(S.Azieb) \\ ${ }^{*}$ Corresponding author
}

\section{To cite this article:}

Samia Azieb, Mahmoud Qudah. The Factors Influencing the Naming Practice in the Algerian Society. American Journal of Art and Design. Vol. 3, No. 1, 2018, pp. 12-17. doi: 10.11648/j.ajad.20180301.13

Received: April 21, 2018; Accepted: May 14, 2018; Published: May 28, 2018

\begin{abstract}
This paper reports on the findings of a study that aims primarily at uncovering the prime factors that direct name choices in Jijel, a province in the Northeast of Algeria. The data were elicited through the administration of a questionnaire to a randomly chosen sample of 150 Algerian males and 150 Algerian females from families in the province of Jijel. The subjects of both genders were taken from three different generations, namely the young generation, the middle-aged generation, and the old generation. Importantly, 50 participants were selected from each single generation. They were asked to identify the factors that they think are prominent in the naming process in their region and to add others if there are any. The findings indicate that the naming practice in the Algerian society has gone through the following path: from a focus on religious, cultural, and family factors in the old generation for both genders, to a more influence coming from the religious factor for both genders in the middle-aged generation. As for the young generation, males' names are still highly influenced by religious beliefs while females' names have shifted towards "trendy or fashionable" ones.
\end{abstract}

Keywords: Algerian Society, Factors, Jijel Province, Naming Practice

\section{Introduction}

The study of names- known as onomastics- and the factors that influence personal name choices-anthroponomastics-in a plethora of societies has been a perennial theme of discussion amongst semanticists from time immemorial. It has also been acted on by different philosophers and anthropologists. Such an interest in the study of names seems to stem, on the one hand, from the fact that humans have tended to use names from the dawn of mankind and, on the other hand, from the fact that naming practices vary enormously across the globe and many things are taken into account when parents choose a name for their newborn children.

Broaching the topic of the factors governing naming individuals necessitates giving heed to the concept of "names". To begin with, Oxford Advanced Learner's Dictionary considers a name as "A word or set of words by which a person or thing is known, addressed, or referred to". Moreover, Saeed [13] states that "names after all are labels for people, places, etc. and often seem to have little other meaning". In a similar vein, Baltes [2] maintains that "the word 'name' itself is often used in the study of language as a designation for any noun... The essence of semantics is naming (Lyons 1977) as names are given to objects or ideas to refer to or to signify the concepts around us". A simpler definition for the word 'name' is provided by Encyclopaedia Britannica [6]: "a word or a group of words that suggest a certain entity whether it's a real or fictitious".

Other attempts to define "name" have yielded other statements by other researchers. For instance, Guma [7] intimates that "Names are more than a 'word' or words by which a person, animal, place or thing is known, and does not fundamentally connote designation, reputation, identification, or separation of one individual from the other per se". He goes even further to claim that names "embody individual or group social experiences, social norms and values, status roles and authority, as well as personality and individual attributes". Moreover, personal names are a sub-component 
of the umbrella term "proper nouns", sometimes referred to as proper names. These terms are inherently designators of individuality (Ullmann, as cited in Baltes, [2]). Interestingly, "a proper name applies uniquely to the possessor of a particular 'individual quality"” (Cumming, [5]).

Evidently, scholars took cognizance of the importance of probing into personal names owing to their ability "to distinguish one individual from another. Since man first began walking on the earth, each individual has had a distinctive name" (Clark, [4]). Names also serve to indicate gender, marital status, birthplace, nationality, ethnicity, religion, and position within a family or even within a society. Above all, names are part and parcel of human life in the sense that "wherever there are people there are names, since names are and have always been part of human life" (Hagstom, [9]). Therefore, "choosing a name for someone else is a very important task" (Watts, [15]).

A myriad of theories which tried to account for names by providing intriguing but only partial explanations have been developed and discounted, indicating that this field is still fraught with controversy. In a nutshell, among the theoretical frameworks which purport that understanding the meaning of a name is inextricably linked to associating it with one or more descriptions is the so-called "the description theory", spearheaded by Russell (1967), Frege (1980), and Searle (1958). Under the banner of this theory, "a name is taken as a label or shorthand for knowledge about the referent, or in the terminology of philosophers, for one or more definite descriptions" (Saeed, [13]). In addition, the "causal theory" was propounded by Devitt and Sterenly (1987) and hanged on the ideas of Kripke (1980) and Donnellan (1972). Such a theory revolves around the main thrust that names are "socially inherited or borrowed" (Saeed, [13]).

It is worth noting that naming- a universal process- is linked to a number of factors which govern it in one way or another. These factors could be, for example, religious, cultural, or social. The choice of names could even reflect a political ideology. According to Pappas [12], the parents' political ideology in the USA, liberal or conservative, can be seen through their choice of their children's names. In this regard, the sounds of liberal and conservative names vary. Parents in liberal neighborhoods are more likely to opt for softer, more feminine sounds, such as "L", and soft-A endings for their babies' names, such as Liam, Ely, Leila, Ella and Sophia. Conservative parents, however, tend to pick names with more masculine-sounding K's, B's, D's and T's, such as Kurt. Indubitably, the influencing factors upon the naming practice in the Algerian society are worthy of investigation. Therefore, to see clearly into the issue under scrutiny, the present study raises two main research questions:

1. What are the factors that direct the choice of males' and females' names in the Algerian society over the old, the middle-aged, and the young generations?

2. What are the main differences and similarities between the factors that affect naming both males and females over the three selected generations?

\section{Literature Review}

The study of names has been the focal point of attention of different studies and research works (Zubatsky, 1996; Singerman, 2001; Lawson, 2003). However, as for the area of the factors that influence offsprings' name choices, prior accounts of it are not abundantly available. This remains a current gap in the existing research on such an intriguing topic. Thus, studies which touch upon the nexus between the naming practice and the factors impacting on it need to be brought into prominence.

Handler and Jacoby [10] inquired into Barbados slave names in order to unveil the principles and significance of naming practices among North American and British Caribbean slaves in general, and on Barbados plantations in particular. A meticulous analysis of plantation slave lists revealed that slaves had some influence on the names by which their children were identified in that the child's parents, especially the mother, exercised the largest role in naming, although the role of grandparents could not be overlooked as well. Moreover, there was no unitary system of naming, either over the approximately two centuries of slavery in Barbados or at particular times during that period.

Likewise, Lawson [11] attempted an investigation of the religious, patriotic, and ethnic factors involved with names and naming in Russia, Latvia, Lithuania, and Azerbaijan. In particular, he tried to determine whether there were similar patterns of religious and patriotic names in Russia and some of the countries that were under its control from the Czarist period untill 1990. The results of such a study indicated that there was a decline in religious names for both sexes from the Czarist period to the end of the interviewing period (1990) except in the case of Latvia where men showed an increase. On the other hand, patriotic names were at relatively low levels during the Czarist period except for the Latvians who were a bit higher. In addition, the Baltic countries had a rise in patriotic names during their period of independence but rose to a much higher level with Russian occupation. As for Russia, it showed an increase in patriotic names after the Revolution. Azerbaijan, under the Czars, however, had no patriotic names but showed a steady increase after the communists took over in 1920.

Following similar lines of inquiry, Gureckis and Goldstone [8] examined how children's names are opted for in the United States by reliance on a historical database of the names given to children over the last century. They came up to the conclusion that naming choices are primarily influenced by both the frequency of a name in the general population, and by its momentum in the recent past in the sense that names which are growing in popularity are chosen at the expense of others.

Clark [4] set light on the naming process in different regions of the United States like Kansas and New Yorkin order to find out whether there is a significant difference between the names chosen for both males and females in those regions with a particular focus on the most popular ones during the birth years of 1976 and 1997. Importantly, he 
was able to identify that in the United States, certain males' names were consistently the names of choice in 1976 and 1977 like "Michael". As for females' names, there seemed to be no noticeable difference between the studied regions of the United States during those years. However, the researcher did not investigate thoroughly why those choices of males' and females' names were made.

Bramwell [3] conducted a cross-cultural study of the naming practices of several diverse communities in Scotland, United Kingdom. He aimed chiefly at comparing and contrasting the personal naming systems of a number of indigenous and immigrant communities whose social and linguistic contexts were different. Particularly, he investigated the links between personal names, social change, cultural contact, and linguistic systems.

Virkkula [14] explored reasons for first name choices for children by drawing data from two different places namely: Zagreb, the capital of Croatia, and Sofia, the capital of Bulgaria. The analysis of both data sets showed that respondents belonging to both categories indicated that they were most concerned with choosing a name they thought was beautiful. After the beauty of the name factor, Zagreb respondents opted for other factors including the meaning of the name, strategies related to national and international positioning, and traditionality of the name. As for Sofia respondents, the most frequently cited reasons were commemoration of relatives, the meaning of the name, whether the name was hereditary, and similarity of the name to other names in the family.

In a more recent piece of research, Al-Qawasmi and AlAbed Al-Haq [1] studied newborn names in Jordan from a sociolinguistic perspective. Their study attempted to detect the difference in naming newborns in Jordan from the seventies to 2015 due to the result of some factors that may have affected the Jordanian society, whether historical, religious and/or social. The data were obtained from the Civil Status Department and the Department of Statistics. They consisted of names of both sexes during the time period from the seventies until the early year of 2015. A quantitative analysis of the data revealed that there is a clear change in the choice of newborn names-male and female-in Jordan, whether a change in sounds or in morphemes. In specific, names during the seventies were strongly linked to the culture and the values, religious or social, which the people believed in. During the eighties and nineties, names were associated with certain social values, however, some names were shown to be affected by urbanization or modernization. And with the beginning of 2000 up to 2015, people's directions towards naming newborns changed due to the advent of globalization, associating with development and urbanization, and moreover, the influence of different cultures on the Jordanian community.

\section{Methodology}

To show that the assumption made in this study is built on solid ground and to accomplish the research aims, a questionnaire was developed and administered to a randomly chosen, gender-balanced sample of 300 individuals who belong to different families in the province of Jijel. These were divided into three generations depending on their age: The Young Generation (1 day- 19 Years), The Middle-aged Generation (20Ys-39 Ys), and The Old Generation: 40 Ys and above). Importantly, 50 informants were taken from each generation for both genders. At this juncture, it is worth nothing that as for very young respondents, their parents answered the questions in the questionnaire on their behalf. That is, they provided the researchers with the factors that lead them to give their children a given name.

\section{Results and Discussion}

The findings of the study are presented and discussed in three sub-sections: (1) The Analysis of Females' Data, (2) The Analysis of Males' Data, and (3) Contrasting Females' and Males' Data over the Three Selected Generations.

\subsection{The Analysis of Females' Data}

Under this heading, the researchers present and discuss the data that are linked to female respondents. Frequency tables and percentages were established to see which factors affect females' naming practice in Jijel and the extent to which their influence increases or decreases over the old, middle-aged, and young generations respectively. Tables 1 through 3 are a case in point.

\subsubsection{The Old Generation}

The findings of this study show that the naming of Algerian females who belong to the old generation (40 Ys and above) is affected by a plethora of factors, be them religious, cultural, or family factors. Table 1 below discloses the needed frequencies and percentages.

Table 1. Factors Affecting the Choice of Algerian Females' Names in the Old Generation.

\begin{tabular}{lll}
\hline Factors & Frequency & Percentage (\%) \\
\hline Religious & 6 & 12 \\
Political & 0 & 0 \\
Family & 17 & 34 \\
Historical & 0 & 0 \\
Cultural & 14 & 28 \\
Musicality of the name & 4 & 8 \\
No Specific factors & 4 & 8 \\
Other factors & 5 & 10 \\
Total & 50 & 100 \\
\hline
\end{tabular}

Clearly, the factor with the highest frequency is the cultural one: 14 (28\%) (examples of names: Akila, Fatiha, and Fadila). This is followed by the family factor with a frequency equals to 12 (24\%) (examples of names: Nowara, Rachida, Sakina). In addition, 6 respondents (12\%) opted for the religious factor to be the one behind the choice of the names that they were given (Eg: Aicha and Khadija). Interestingly, the musicality of the name factor and the no specific reason for naming factor received the same frequency: 4 (8\%) (Eg: Cherifa and Dahbiya). The lowest 
percentage is occupied by the political and historical factors with a 0 frequency.

Some informants stated that two other factors namely: the weather conditions in which the girl was born $(4 \%)$ (for example the name Talja "snow") and the season of birth as well (6\%) (the name Rabia "spring") were the reasons behind the names that they were given.

\subsubsection{The Middle-Aged Generation}

Data bearing on the factors that directed the naming of middle-aged females in Jijel are manifested in Table 2 below:

Table 2. Factors Affecting the Choice of Algerian Females' Names in the Middle-aged Generation.

\begin{tabular}{lll}
\hline Factors & Frequency & Percentage (\%) \\
\hline Religious & 16 & 32 \\
Political & 0 & 0 \\
Family & 9 & 18 \\
Historical & 2 & 4 \\
Cultural & 4 & 8 \\
Musicality of the name & 7 & 14 \\
No Specific Factors & 5 & 10 \\
Other Reasons & 7 & 14 \\
Total & 50 & 100 \\
\hline
\end{tabular}

The responses of female informants belonging to the middle- aged generation, as shown in the above table, are indicative of the fact that the religious factor had the highest frequency: 16 with a percentage of $32 \%$ (Eg: Fatima, Aicha, Zeineb). Next, the family factor had a frequency of $9(18 \%)$ (Eg: Noura, Hafsa, Nahla, and Latifa). The third frequency was recorded for the musicality of the name factor: 7 (14\%). 5 respondents, however, i.e. $(10 \%)$ chose the factor of no specific reason for naming (Eg: Mona, Mofida, Karima). Lower percentages were occupied by the cultural, historical and political factors with the values of $8 \%, 4 \%$ and $0 \%$ respectively.

Some respondents (7) suggested that the names they were given at birth were governed by the "meaning of the name" factor like Nour Elhoda, and Nour Eliman.

\subsubsection{The Young Generation}

Table 3 shows the frequencies and the percentages which indicate the extent to which each factor affected the naming of Algerian females in the young generation:

Table 3. Factors Affecting the Choice of Algerian Females' Names in the Young Generation.

\begin{tabular}{lll}
\hline Factors & Frequency & Percentage (\%) \\
\hline Religious & 10 & 20 \\
Political & 0 & 0 \\
Family & 5 & 10 \\
Historical & 1 & 2 \\
Cultural & 2 & 4 \\
Musicality of the name & 7 & 14 \\
No Specific Reasons & 0 & 0 \\
Other Reasons & 25 & 50 \\
Total & 50 & 100 \\
\hline
\end{tabular}

Table 3 indicates that dealing with the factors affecting the choice of females' names belonging to the young generation seems to have classified those factors (apart from the other reasons provided by the respondents) according to their frequencies and percentages from the highest to the lowest as follows: the religious factor $(20 \%)$ (Eg: Halima, and Amina), the musicality of the name (14\%) (Eg: Lina, and Dina), the family factor $(10 \%)$ (Eg: Sara, and Marwa), the cultural factor (4\%) (Eg: Fatwa, and Fadwa), the historical factor (2\%) (Eg: Hassiba, Malika, and Wrida) and, finally, the political factor $(0 \%)$.

The respondents added two intriguing factors. The first one is "the meaning of the name factor" (10\%) (Rawnak, and Najah). The second one is the "trendy or fashionable names" factor. It received the highest frequency and percentage 20 (40\%) (Eg: Norhan, Roa, Arij, and Lojayn).

\subsection{The Analysis of Males' Data}

The following three tables $(4,5$, and 6$)$ show the results of the frequencies and percentages that are related to the factors that have an impact on males' name choices in the Algerian society over three selected generations: the old generation, the middle-aged generation, and the young generation.

\subsubsection{The Old Generation}

Based on the gathered data, the researchers were able to identify the factors that played the most important role in naming male individuals who belong to the old generation. Table 4 is in order here:

Table 4. Factors Affecting the Choice of Algerian Males' Names in the Old Generation.

\begin{tabular}{lll}
\hline Factors & Frequency & Percentage (\%) \\
\hline Religious & 12 & 24 \\
Political & 3 & 6 \\
Family & 11 & 22 \\
Historical & 1 & 2 \\
Cultural & 13 & 26 \\
Musicality of the name & 3 & 6 \\
No Specific Reasons & 3 & 6 \\
Other Reasons & 4 & 8 \\
Total & 50 & $100 \%$ \\
\hline
\end{tabular}

Table 4 casts light on the fact that a considerable number of respondents (13) reported that the cultural factor (Eg. Ammar, Bachir, and Tahar) governed their naming practice. Other factors including the religious (Eg. Ahmed, Mohammed, and Abdellah), and the family factors (Eg. Azzedine, Hocine, and Mobarak) received almost the same frequencies, with $12(24 \%)$ and $11(22 \%)$ respectively. Identical frequencies were reserved for the three factors: political, musicality of the name, and the no reason factor: 3 $(6 \%)$. The historical factor was reported to receive the lowest frequency and percentage: $1(2 \%)$.

One more factor was furnished by the respondents namely: the month in which the male was born: (8\%) (Chaabane, and Ramadan).

\subsubsection{The Middle-Aged Generation}

The analysis of the data under investigation provides evidence for the fact that the religious factor is the one that 
greatly directed the naming practice of males that belong to the middle-aged generation. Table 5 is a case in point:

Table 5. Factors Affecting the Choice of Algerian Males' Names in the Middle-aged Generation.

\begin{tabular}{lll}
\hline Factors & Frequency & Percentage (\%) \\
\hline Religious & 19 & 38 \\
Political & 0 & 0 \\
Family & 8 & 16 \\
Historical & 8 & 16 \\
Cultural & 2 & 4 \\
Musicality of the name & 4 & 8 \\
No Specific Reasons & 4 & 8 \\
Other Reasons & 5 & 10 \\
Total & 50 & $100 \%$ \\
\hline
\end{tabular}

It is noticeable that the highest percentage was occupied by the religious factor: $38 \%$ with a frequency of 19 (Eg. Abderrahim, Mohammed, and Abdelaziz). In a similar vein, family and historical factors (Eg. Bakir, Alarbi, and Ahmed) seemed to play a significant role in choosing the names of males in the Algerian society: $8(16 \%)$. Two other factors namely: the musicality of the name and the no specific reason factor received equal frequencies: $4(8 \%)$. The lowest percentages were recorded for both the cultural and the political factors, with the frequencies of $2(4 \%)$ and $0(0 \%)$ respectively.

It should be noted that 2 participants (4\%) added "the meaning of the name" as the factor governing male naming practice (Baha Dine, and Fatah) while 3 of them (6\%) stated that their names were opted for "because their mothers used to love persons with the same names that they were given" (Mahdi, Farid, and Faress).

\subsubsection{The Young Generation}

In relation to the young generation, the following findings in Table 6 were attested:

Table 6. Factors Affecting the Choice of Algerian Males' Names in the Young Generation.

\begin{tabular}{lll}
\hline Factors & Frequency & Percentage (\%) \\
\hline Religious & 21 & 42 \\
Political & 0 & 0 \\
Family & 5 & 10 \\
Historical & 1 & 2 \\
Cultural & 0 & 0 \\
Musicality of the & 7 & 14 \\
name & 2 & 4 \\
No Specific Reasons & 14 & 28 \\
Other Reasons & 50 & $100 \%$ \\
Total &
\end{tabular}

Considering table 6 , one can notice that the religious factor governing naming males remained in the lead by receiving again the highest percentage: $42 \%$ (Eg. Mohammed, Yahia, and Ayoub). Other factors were classified according to their effect on males' naming practice from the highest to the lowest as follows: the musicality of the name factor (14\%), the family factor $(10 \%)$, the no specific reason factor $(4 \%)$, the historical factor $(2 \%)$, the political and the cultural factors $(0 \%)$.
Two more factors were supplied by the respondents: "the meaning of the name factor" which received a percentage of $(8 \%)$, and the "recent or trendy name factor" received $(20 \%)$, thus being the second high-ranked factor for choosing males' names in the young generation group.

\subsection{Contrasting Females' and Males' Data over the Three Generations}

\subsubsection{Similarities}

The analysis of the data reveals the following similarities between the factors affecting males' and females' naming practice over the old, middle-aged, and young generations in the Jijilian society in Algeria:

1. In the old generation, the naming of both males and females is highly affected by religious beliefs, the culture of the place in which the person lives in addition to naming them after their relatives- what is called namesake-. For instance, firstborn boys are usually named after their father or paternal grandfather (sometimes deceased ones) and firstborn girls are named after their paternal grandmother.

2. The primacy is given for the religious factor in the middle-aged generation for both males and females. This is coupled by a remarkable decrease in the role played by the cultural factors for both genders and the addition of "meaning of the name factor" by the participants in both groups.

3. As far as the young generation is concerned, "the meaning of the name" factor is added for both genders, and the religious factor is still significant though with different degrees. Moreover, the cultural factor plays a less important role for the choice of both males' and females' names.

\subsubsection{Differences}

Despite the above-mentioned similarities, one salient difference is attested:name choice for Jijilian Algerian females has gone through the following path: there is a focus on the cultural and family factors and to some extent on the religious one in the old generation, then there was a shift to the religious factor as the most prominent one in the middleaged generation. Intriguingly, however, the winning factor in the young generation for females is the "trendy or fashionable names factor". On the other hand, the males' naming practice has witnessed almost the same thing in the old and middle-aged generation with the salient difference apparent in the fact that in the young generation, males 'names are still subject to the influence of the religious factor (preferring the names of the Prophets) more than females which have shifted almost totally to a preference of trendy or fashionable names.

\section{Conclusion}

Carrying out a thorough analysis of the data in the present study has revealed that the choice of given names in Jijel/ Algeria is influenced by an array of factors which changed throughout three selected generation: The Old Generation, The Middle-aged Generation, and The Young Generation. In 
other words, when choosing names, parents often consider those factors depending, of course, on the generation being considered and the gender as well. Axiomatically, the major difference between the factors influencing the choice of males' and females' names is obvious in the young generation in that, for males, the religious belief still has a significant influence on their names' choice while females' names have taken a brand new direction: "fashionable names", the names of celebrities resulting from the influence of the mass media in general. After all, we are consumers of names, names are part and parcel of our lives and we need to know why we were given the names we currently bear.

\section{Recommendations}

Hopefully, the present study will pave the road for subsequent studies about a highly important topic like the one in hand. Therefore, follow up studies on the factors influencing the naming practice in other regions of Algeria are recommended so as to gain new insights on the extent to which they differ from one region to another and from one gender to another. In addition, cross-cultural studies in the same area are recommended.

\section{References}

[1] Al-Qawasmi, A., \& Al-Abed Al-Haq, F. (2016). A Sociolinguistic study of choosing names for newborn children in Jordan, International Journal of English Linguistics, 6 (1), 177-186.

[2] Baltes, P. (1991). Semantic variation in the connotations of personal names. DLLS Proceedings, Purdue University.

[3] Bramwell, E. (2012). Naming in society: A cross-cultural study of five communities in Scotland. (Published doctoral thesis). University of Glasgow.
[4] Clark, S. (2010). From Adam to Zara: Popularity of names in regions of the United States. FAST-US-7 (TRENAK15) United States Popular Culture (Hopkins), Department of Translation Studies, University of Tampere.

[5] Cumming, J, S (2007). Proper nouns (Published doctoral thesis). The State University of New Jersy.

[6] Encyclopedia Britannica. (1926). London: The Encyclopedia Britannica Inc.

[7] Guma, M. (2001). The cultural meaning of names among Basotho of Southern Africa: A historical and linguistic analysis, Nordic Journal of African Studies, (3), 265-279.

[8] Gureckis, T., \& Goldstone, R. (2009). How you named your child: Understanding the relationship between individual decision making and collective outcomes, Topics in Cognitive Science, (1), 651-674.

[9] Hagstom, C. (2012). Naming me, naming you. Personal names, online signatures and cultural meaning, Oslo Studies in Language, 4(2), 81-93.

[10] Handler, J., \& Jacoby, J. (1996). Slave names and naming in Barbados, 1650-1830, The William and Mary Quarterly, Third Series, 53 (4), 685-728.

[11] Lawson, D (2008). Religious, patriotic, and ethnic factors involved with names and naming in Russia, Latvia, Lithuania, and Azerbaijan. State University of New York at Fredonia.

[12] Pappas, S. (2013). Baby names reveal parents' ppolitical ideology. Live Science Contributor.

[13] Saeed. J (1999). Semantics. London: Blackwell publishing.

[14] Virkkula, J. (2014). First name choices in Zagreb and Sofia. (Published doctoral thesis). University of Helsinki, Finland.

[15] Watts, N. (2008). The art of baby nameology: Explore the deeper meaning of names for your baby. Illinois: Sourcebooks, Inc. 